\title{
Shadows of Kerr Black Holes with Scalar Hair
}

\author{
Pedro V. P. Cunha, ${ }^{1,2}$ Carlos A. R. Herdeiro, ${ }^{2}$ Eugen Radu, ${ }^{2}$ and Helgi F. Rúnarsson ${ }^{2}$ \\ ${ }^{1}$ Departamento de Física, Universidade de Coimbra, 3004-516 Coimbra, Portugal \\ ${ }^{2}$ Departamento de Física da Universidade de Aveiro and CIDMA, Campus de Santiago, 3810-183 Aveiro, Portugal
}

(Received 4 September 2015; revised manuscript received 29 September 2015; published 16 November 2015)

\begin{abstract}
Using backwards ray tracing, we study the shadows of Kerr black holes with scalar hair (KBHSH). KBHSH interpolate continuously between Kerr BHs and boson stars (BSs), so we start by investigating the lensing of light due to BSs. Moving from the weak to the strong gravity region, BSs-which by themselves have no shadows-are classified, according to the lensing produced, as (i) noncompact, which yield not multiple images, (ii) compact, which produce an increasing number of Einstein rings and multiple images of the whole celestial sphere, and (iii) ultracompact, which possess light rings, yielding an infinite number of images with (we conjecture) a self-similar structure. The shadows of KBHSH, for Kerr-like horizons and noncompact BS-like hair, are analogous to, but distinguishable from, those of comparable Kerr BHs. But for non-Kerr-like horizons and ultracompact BS-like hair, the shadows of KBHSH are drastically different: novel shapes arise, sizes are considerably smaller, and multiple shadows of a single BH become possible. Thus, KBHSH provide quantitatively and qualitatively new templates for ongoing (and future) very large baseline interferometry observations of BH shadows, such as those of the Event Horizon Telescope.
\end{abstract}

DOI: 10.1103/PhysRevLett.115.211102

PACS numbers: 04.20.-q, 04.70.Bw, 04.80.Cc

Introduction.-One hundred years after general relativity (GR) was formulated, we finally face a realistic prospect of testing one of its most dramatic consequences: black holes (BHs). The evidence for astrophysical BHs, gathered for over half a century, has built a strong case [1], but it could not confirm the existence of event horizons, the defining property of BHs. The near future promises to open up a new channel of observation-gravitational waves - and deliver electromagnetic measurements of unprecedented precision, hopefully clarifying this central issue [2].

A particularly exciting prospect is the use of very large baseline interferometry (VLBI) techniques to resolve the angular scale of the event horizon for some supermassive $\mathrm{BH}$ candidates and determine the corresponding $\mathrm{BH}$ shadow $[3,4]$. Its observation would probe the spacetime geometry in the vicinity of the horizon and consequently test the existence and properties of the latter [5]. It is therefore timely to study $\mathrm{BH}$ models that yield phenomenological deviations from the paradigmatic GR $\mathrm{BH}$, described by the Kerr metric.

One approach is to parametrize families of metric deviations from Kerr [6-11]. Another approach is to use exact solutions of GR (or generalizations thereof) yielding deviations from Kerr (e.g., Refs. [12-14]). Exact solutions with physically reasonable and astrophysically plausible matter sources, however, are scarce, but Kerr BHs with scalar hair (KBHSH) [15] are arguably one such model. These are exact solutions of Einstein's gravity minimally coupled to a massive complex scalar field, and they interpolate between Kerr BHs and gravitating solitonsboson stars (BSs) [16]—suggested as dark matter candidates (in the Newtonian limit) and BH mimickers [17-19].
In this Letter we show that the shadows of KBHSH are distinguishable, or even drastically different, from those of Kerr BHs, and can thus yield new templates for the ongoing and future VLBI searches [20] of BH shadows.

The solutions.-KBHSH can be expressed by a stationary and axisymmetric line element, in spheroidal coordinates $(t, r, \theta, \phi)[15,21]$, together with the (mass $\mu$ ) scalar field $\Psi=\phi(r, \theta) e^{i(m \varphi-w t)}$, where $w$ is the frequency and $m \in \mathbb{Z}^{+}$is the azimuthal harmonic index. The metric functions and $\phi$ are determined numerically by solving five coupled, nonlinear partial differential equations [21]. For $m \geq 1$, both BSs and KBHSH solutions can be obtained. For $m=0$, spherical BSs exist. The space of solutions for $m=0,1$ is summarized in Fig. 1. BSs exist for a limited range of $w$ along spiraling curves. KBHSH exist inside an open set, bounded by the $m=1 \mathrm{BS}$ curve, a set of Kerr BHs, and the set of extremal KBHSH.

Setup.-Our goal is to compute photon geodesics in the above geometries and obtain, at a given observation point, the distorted apparent sky, due to the gravitational lensing of BSs, as well as the shadow, when a horizon is present. To interpret the patterns obtained, we divide the "celestial sphere" light source into four quadrants, each painted with a different color. On top of these, a grid of constant longitude and latitude (black) lines is introduced, with adjacent lines separated by $10^{\circ}$; Fig. 2 (left). This setup mimics closely the one in Ref. [22].

The observer, henceforth denoted as $\mathcal{O}$, is placed inside the celestial sphere at an off-centered position to be specified below, and its viewing angle captures $\sim 100^{\circ}$ of that sphere; see Fig. 2 (right). The point on the celestial sphere immediately in front of $\mathcal{O}$ (dubbed $F$ ) lies at the 


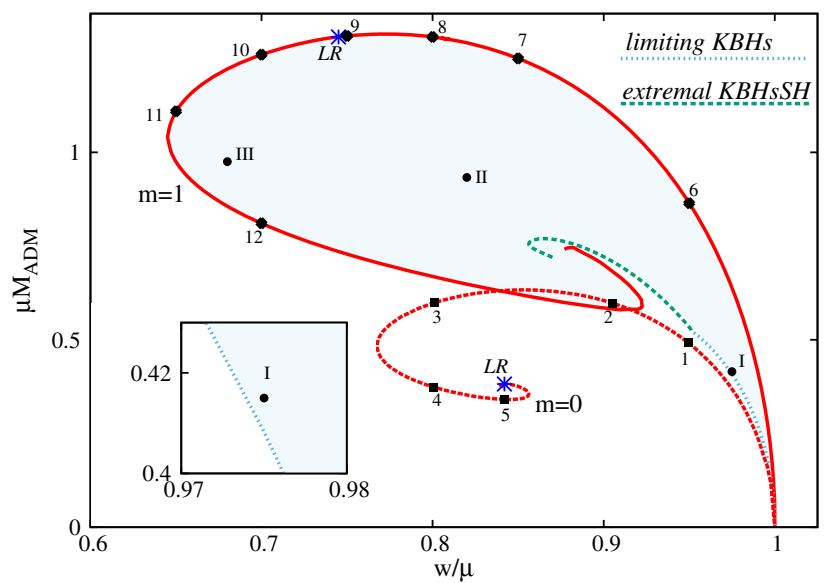

FIG. 1 (color online). BS solutions with $m=0,1$ (dashed and solid red lines) in an ADM mass $M_{\mathrm{ADM}}$ vs scalar field frequency $w$ diagram (in units of $\mu$ ). For $m=1$, KBHSH exist within the shaded region. Points 1-12 (I-III) correspond to the BSs (KBHSH) exhibited below.

intersection of the four colored quadrants, and it is marked with a white dot.

From the $\mathcal{O}$ 's position we span its viewing angle by performing, numerically, backwards ray tracing of $2000 \times 2000$ photon trajectories. We integrate these null geodesics until they either reach a point on the celestial sphere or they hit the BH horizon (when it is present). The latter directions form the shadow [23]. O's image is obtained upon a projection onto its local frame, by a method similar to that in Ref. [22].

Quantitative shadow parameters.-To analyze the shadows obtained below we introduce six parameters, $\left\{D_{C}, D_{x}, D_{y}, \bar{r}, \sigma_{r}, \sigma_{\mathrm{Kerr}}\right\}$, mostly following Ref. [24]. Let $\mathcal{O}$ 's image be parametrized by the Cartesian coordinates $(x, y)$, obtained from multiplying the observation angles by the circumferential radius $R$ [25] at $\mathcal{O}$ 's position. The origin of this coordinate system $O$ points at the (unlensed) white dot on the celestial sphere. The center of the shadow $C$ has $x_{C}=\left(x_{\max }+x_{\min }\right) / 2$, where $x_{\max }$ and $x_{\min }\left(y_{\max }\right.$ and $\left.y_{\min }\right)$ are, respectively, the maximum and minimum abscissae (ordinates) of the shadow's edge. Because of reflection
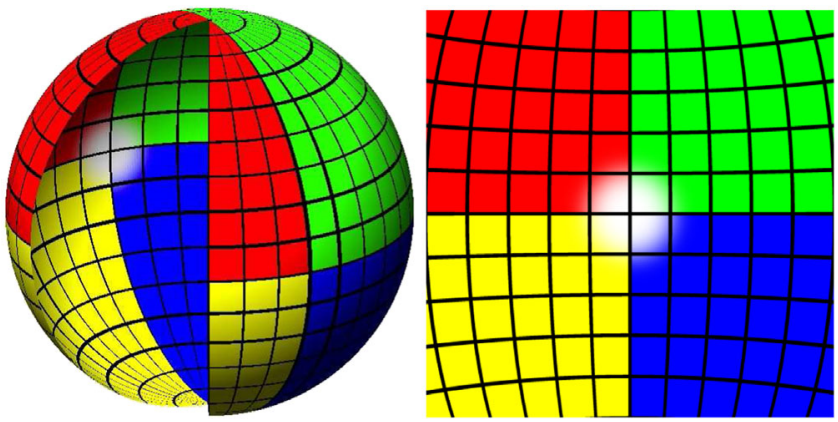

FIG. 2 (color online). Left: The full celestial sphere. Right: The viewing angle of the off-centered observer $(\mathcal{O})$. symmetry for observations on the equatorial plane, $y_{C}=0 . C$ and $O$ need not coincide; the displacement $D_{C} \equiv\left|x_{C}\right|$ measures their difference. The width and height of the shadow are, respectively, $D_{x} \equiv x_{\max }-x_{\min }$ and $D_{y} \equiv y_{\max }-y_{\min }$.

A generic point $P$ on the shadow's edge is at a distance $r \equiv\left[y_{P}^{2}+\left(x_{P}-x_{C}\right)^{2}\right]^{1 / 2}$ from $C$. Let $\alpha$ be the angle between the line $\overline{C P}$ and the $x$ axis; the average radius is $\bar{r} \equiv \int_{0}^{2 \pi} r(\alpha) d \alpha / 2 \pi$, and the deviation from sphericity is $\sigma_{r} \equiv\left\{\int_{0}^{2 \pi}[r(\alpha)-\bar{r}]^{2} d \alpha / 2 \pi\right\}^{1 / 2}$. Finally,

$$
\sigma_{K} \equiv \sqrt{\frac{1}{2 \pi} \int_{0}^{2 \pi}\left(\frac{r(\alpha)-r_{\mathrm{Kerr}}(\alpha)}{r_{\mathrm{Kerr}}(\alpha)}\right)^{2} d \alpha}
$$

is the relative deviation from a comparable Kerr $\mathrm{BH}$, either with the same Arnowitt-Deser-Misner (ADM) mass and angular momentum $M_{\mathrm{ADM}}, J_{\mathrm{ADM}}$ or with the same horizon quantities $M_{H}, J_{H}$ (as long as the Kerr bound is not violated for the comparable Kerr BH). These comparable Kerr BHs are denoted below as $\mathrm{Kerr}_{=\mathrm{ADM}}$ and $\mathrm{Kerr}_{=H}$, respectively. $C$ is made to coincide for both BHs in (1).

Lensing by spherical BSs. - We first look at the lensing due to spherically symmetric BSs; see Fig. 3 . We set $\mathcal{O}$ on the equator and always at $R=22.5 / \mu$, for the different $\mathrm{BS}$ solutions. Throughout, we take $G=1=c$ and in the following, unless otherwise stated, $\mu=1$.

Starting from vacuum (i.e., $w=1$ ) we find a set of noncompact $\mathrm{BSs}$, for which any meaningful effective radius is large as compared to the corresponding Schwarzschild radius. These are "weak gravity" solutions and the corresponding lensing is illustrated by the BS with $w_{1}^{(b 1)}=0.95$ [26] in Fig. 3, where only a small distortion of the background is observed. Moving further along the

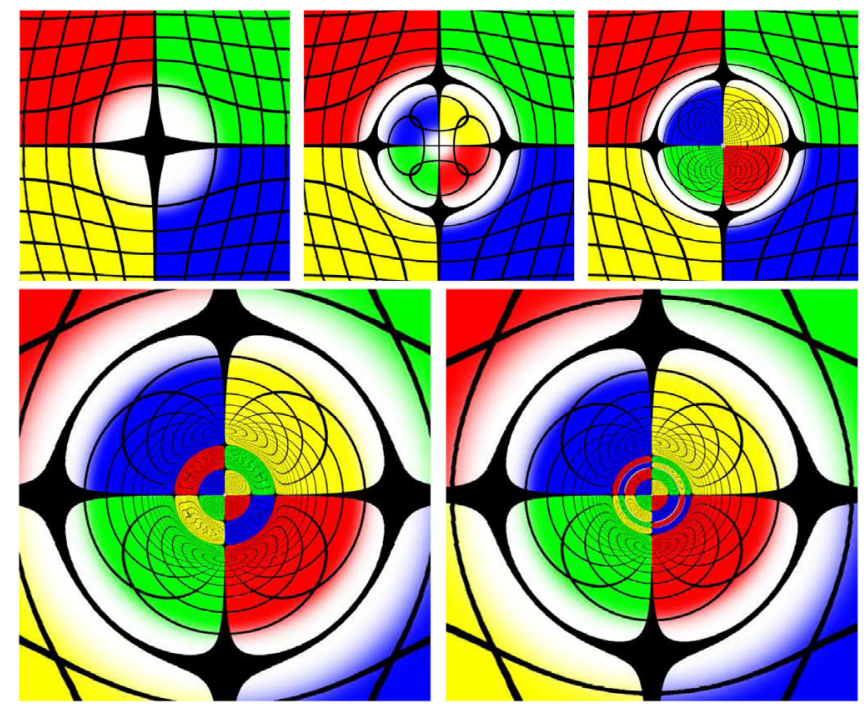

FIG. 3 (color online). Lensing by spherical BSs. From left to right, top $w_{1,2,3}^{(b 1)}=0.95 ; 0.9 ; 0.8$ and bottom $w_{4,5}^{(b 2)}=0.8 ; 0.84$ (zoomed). 
spiral, an Einstein ring appears at $w_{\mathrm{ER} 1}^{(b 1)} \simeq 0.94$. The Einstein ring is formed by the lensing of $F$, and it encloses two inverted copies of a region around $F$. This is illustrated in Fig. 3 using a BS with $w_{2}^{(b 1)}=0.9$. The appearance of the first Einstein ring defines the transition from noncompact to compact BSs.

Moving further along the spiral the region duplicated inside the Einstein ring becomes an increasingly larger part of the celestial sphere, as can be observed for the BS with $w_{3}^{(b 1)}=0.8$ in Fig. 3. Shortly after the spiral's first backbending, at $w_{\mathrm{BB} 1}^{(b 1 \rightarrow b 2)} \simeq 0.767$ (cf. Fig. 1), the full celestial sphere becomes duplicated, starting at the BS with $w_{\mathrm{ER} 2}^{(b 2)} \simeq 0.77$. At and beyond this point, two further Einstein rings emerge-now corresponding to lensings of the point immediately behind the observer (dubbed $B$ ). This is illustrated by the bottom left panel in Fig. 3, for a BS with $w_{4}^{(b 2)}=0.8$. In between these two new Einstein rings, further pairs of Einstein rings can be seen to appear, progressively, further inside the spiral. The first such pair is illustrated in Fig. 3 for $w_{5}^{(b 2)}=0.84$. Each new pair of Einstein rings corresponds to images of either $F$ or $B$, in an alternating fashion, and to a further complete copy of the full celestial sphere.

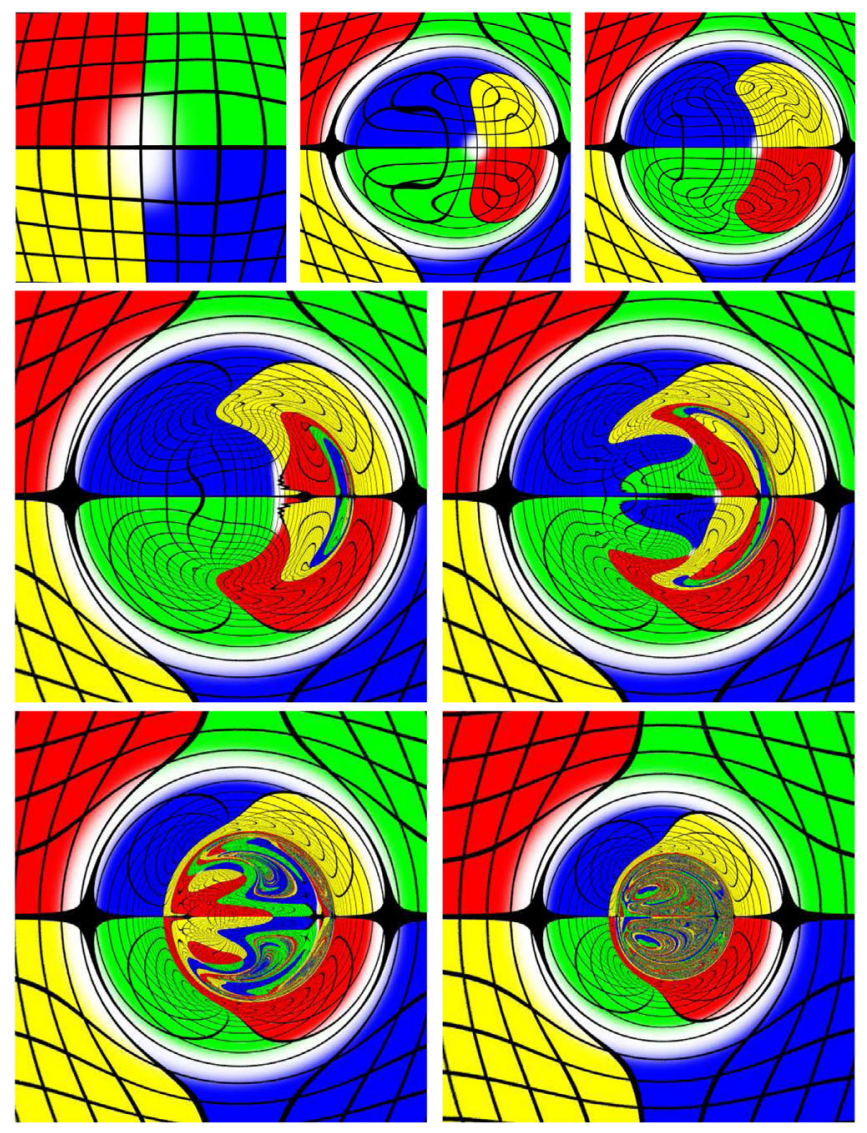

FIG. 4 (color online). Lensing by rotating BSs with $m=1$. From left to right, top $w_{6,7,8}^{(b 1)}=0.95 ; 0.85 ; 0.8$, middle $w_{9,10}^{(b 1)}=0.75 ; 0.7$, and bottom $w_{11}^{(b 1)}=0.65 ; w_{12}^{(b 2)}=0.7$.
An infinite number of copies, and a corresponding selfsimilar structure, is expected to arise when a light ringcorresponding in this case to a photon sphere-appears [27], marking the transition from compact to ultracompact [28] BSs. This occurs well inside the spiral, on the third branch (after the second backbending), starting at the BS solution with $w_{\mathrm{LR}}^{(b 3)}=0.842$, marked as the blue star light ring (LR) point on the $m=0$ spiral of Fig. 1 .

Lensing by rotating $B S s$. - We now turn to rotating BSs (spin axis pointing up). Starting from vacuum, we again find a region of noncompact $\mathrm{BSs}$, i.e., without multiple images. Two differences, however, with respect to the top left panel of Fig. 3 are an asymmetric lensing, with an amplification of the side rotating away from $\mathcal{O}$, and the slight shift of point $F$ to the left, due to frame dragging; these are illustrated in Fig. 4 for a BS with $w_{6}^{(b 1)}=0.95$. At $w_{\mathrm{ER} 1}^{(b 1)} \simeq 0.92$ an Einstein ring appears, starting the set of
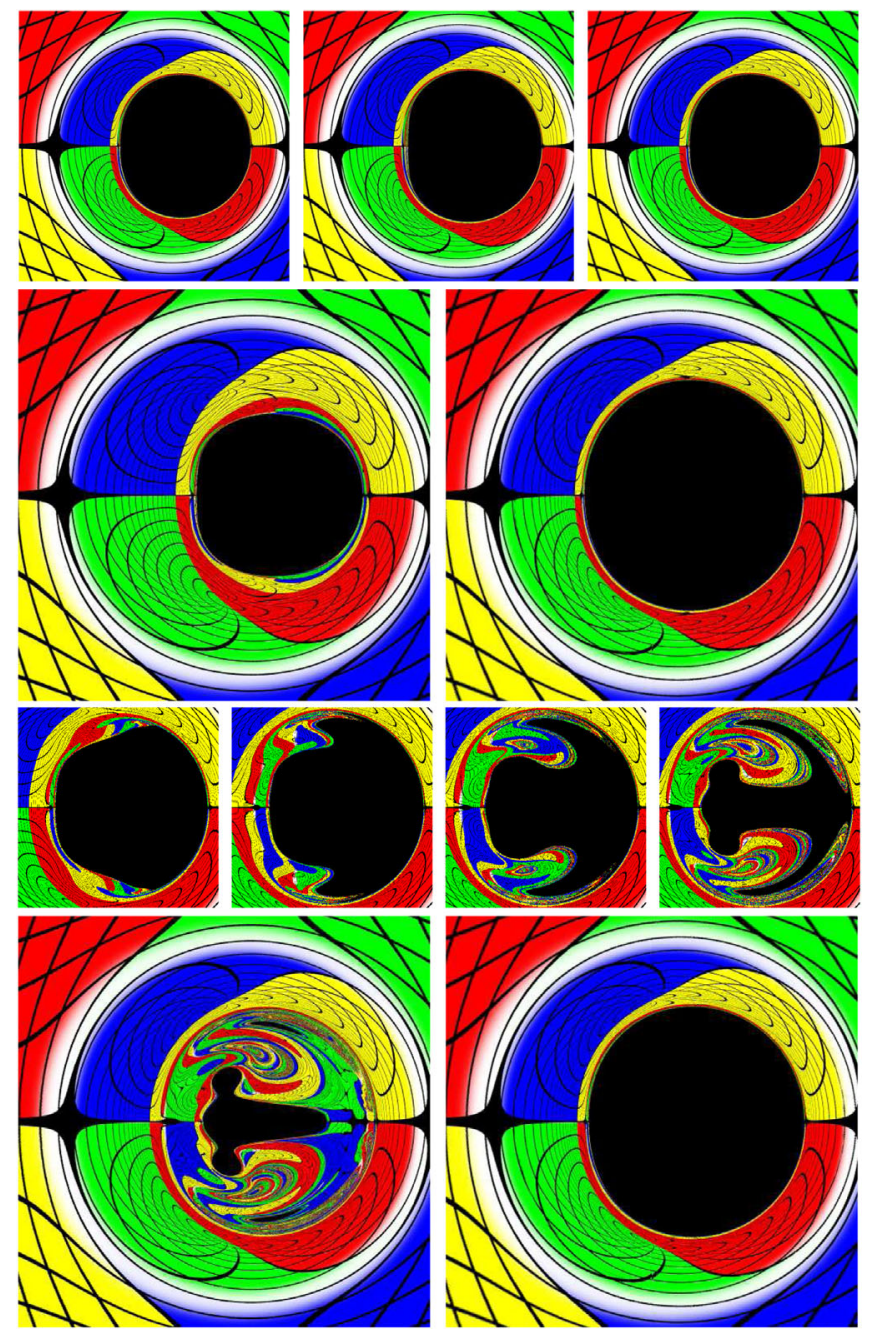

FIG. 5 (color online). From left to right, shadows of (top) configuration I, Kerr $=\mathrm{ADM}$, and $\mathrm{Kerr}_{=H}$, (second row) configuration II and $\mathrm{Kerr}_{=\mathrm{ADM}}$, (third row) transition configurations between II and III (detail), and (bottom) configuration III and Kerr $=$ ADM 
TABLE I. Physical quantities in units of $\mu$, when dimensionful of configurations I-III in Fig. 1.

\begin{tabular}{lcclccccc}
\hline \hline & $M_{\mathrm{ADM}}$ & $M_{H}$ & $J_{\mathrm{ADM}}$ & $J_{H}$ & $M_{H} / M_{\mathrm{ADM}}$ & $J_{H} / J_{\mathrm{ADM}}$ & $J_{\mathrm{ADM}} / M_{\mathrm{ADM}}^{2}$ & $J_{H} / M_{H}^{2}$ \\
\hline I & 0.415 & 0.393 & 0.172 & 0.150 & $95 \%$ & $87 \%$ & 0.999 & 0.971 \\
II & 0.933 & 0.234 & 0.740 & 0.115 & $25 \%$ & $15 \%$ & 0.850 & 2.10 \\
III & 0.975 & 0.018 & 0.85 & 0.002 & $1.8 \%$ & $2.4 \%$ & 0.894 & 6.20 \\
\hline \hline
\end{tabular}

compact rotating BSs. This is the case, in Fig. 4, for the BSs with $w_{7.8}^{(b 1)}=0.85 ; 0.8$. The ring encloses again two inverted copies of part of the celestial sphere, but it is now elliptic and the duplicated image of the side rotating towards the observer is suppressed. The inversion shifts point $F$ to the right.

Further following the spiral, new Einstein "rings" appear, just as in the case of spherical BSs, except that instead of an " $O$ shape," they have a squashed " $D$ shape." An example, for a BS with $w_{9}^{(b 1)}=0.75$, is shown in Fig. 4. Then, a light ring on the equatorial plane appears at $w_{\mathrm{LR}}^{(b 1)} \simeq 0.747$. Beyond this point multiple (presumably infinitely many) images of the celestial sphere arise, with, we conjecture, a fractal structure. This is illustrated by the BSs with $w_{10,11}^{(b 1)}=0.7 ; 0.65$ and $w_{12}^{(b 2)}=0.7$ in Fig. 4.

Shadows of KBHSH.-A KBHSH may be regarded as a BS around (and corotating with) a central horizon. The latter may be non-Kerr-like, i.e., violate the Kerr bound in terms of horizon quantities [29], and the former may have strong lensing effects, as seen above. Consequently, it is expectable that KBHSH with ultracompact BS-like hair and with a non-Kerr-like central horizon will have unfamiliar shadows. This expectation is confirmed in Fig. 5, where we exhibit the shadows and lensing for three examples of KBHSH (together with four "transition" examples) and for comparable Kerr BHs. The three main examples are configurations I-III in Fig. 1, and their physical quantities, in units of $\mu$ when dimensionful, are summarizes in Table I.

In Fig. 5, we have scaled $\mu$ in each case so that $M_{\mathrm{ADM}}$ is the same for all KBHSH presented, and $\mathcal{O}$ sits at $R=15 M_{\mathrm{ADM}}$. Comparing the shadows in this way bears more significance for real observations, where $M_{\mathrm{ADM}}$ of the $\mathrm{BH}$ is fixed by the data.

The top left panel of Fig. 5 shows the shadow of configuration I (shadow I for short). For this KBHSH the "hair" contains only $5 \%$ of the total mass, the "nearby" BSs in Fig. 1 are noncompact, and the central horizon is Kerr-like $\left(J_{H} / M_{H}^{2}<1\right)$. The shadow, albeit qualitatively familiar, is nevertheless distinguishable from that of the $\mathrm{Kerr}_{=\mathrm{ADM}} \mathrm{BH}$, which is exhibited in the top middle panel. The latter is slightly larger and more $D$-like-a characteristic of extremal Kerr BHs. Shadow I turns out to be closer to the one of the $\mathrm{Kerr}_{=H} \mathrm{BH}$, exhibited in the top right panel. This observation can be quantitatively checked: $\sigma_{K}=4.81 \% / 0.52 \%$, taking $\mathrm{Kerr}_{=\mathrm{ADM}} / \mathrm{Kerr}_{=H}$ for the comparable $\mathrm{BH}$; cf. Table II.

New types of $\mathrm{BH}$ shadows, quite distinct from those of Kerr $=$ ADM BHs, appear on the left second and fourth row panels of Fig. 5, corresponding to shadows II and III. In both cases, the central $\mathrm{BH}$ is non-Kerr-like $\left(J_{\mathrm{BH}} / M_{\mathrm{BH}}^{2}>1\right)$. Shadow II is smaller (average radius of $\sim 75 \%$ ) than that of the Kerr $=$ ADM BH. It is also more "square," with a larger normalized deviation from sphericity. Shadow III is remarkably distinct. Its central BH has $J_{H} / M_{H}^{2} \sim 6.2$, allowed by the "heavy" hair that it is dragging (cf. the discussion in Ref. [29]). The lensing of this hair resembles closely that of the ultracompact BS on the bottom left panel of Fig. 4. Interestingly, multiple disconnected shadows of the (single) BH occur: the largest ones (besides the main "hammerlike" shadow) are two eyebrows [30,31], at symmetric positions above and below the main shadow, but we have detected many other smaller shadows, hinting again at a self-similar structure. On the third row of Fig. 5, the shadows of four solutions in

TABLE II. Parameters for Kerr shadows with $A$ are computed from the analytic solution [3]; $\sigma_{K}$ is always computed with respect to such solution. The second line in the table, computed for a Kerr BH generated numerically and using the same ray tracing code as for $\mathrm{KBHSH}$, estimates the numerical error $(\sim 1 \%)$ of the KBHSH shadows.

\begin{tabular}{|c|c|c|c|c|c|c|c|}
\hline & $D_{C}$ & $D_{x}$ & $D_{y}$ & $\bar{r}$ & $\sigma_{r}$ & $\sigma_{r} / \bar{r}(\%)$ & $\sigma_{K}(\%)$ \\
\hline Shadow I & 2.07 & 8.48 & 9.33 & 4.48 & 0.170 & 3.8 & $4.81 / 0.52$ \\
\hline $\mathrm{Kerr}_{=\mathrm{ADM}}$ & 2.30 & 8.73 & 9.76 & 4.67 & 0.217 & 4.64 & 1.21 \\
\hline $\operatorname{Kerr}_{=\mathrm{ADM}} A$ & 2.38 & 8.66 & 9.86 & 4.70 & 0.260 & 5.54 & 0 \\
\hline $\mathrm{Kerr}_{=H} A$ & 2.07 & 8.48 & 9.36 & 4.50 & 0.180 & 3.99 & 0 \\
\hline Shadow II & 2.39 & 7.14 & 6.93 & 3.60 & 0.118 & 3.29 & 25.5 \\
\hline Kerr $_{=\text {ADM }} A$ & 1.79 & 9.32 & 9.86 & 4.82 & 0.103 & 2.15 & 0 \\
\hline Shadow III & 1.79 & 5.30 & 4.67 & 1.63 & 0.838 & 51.3 & 68.1 \\
\hline $\mathrm{Kerr}_{=\mathrm{ADM}} A$ & 1.92 & 9.22 & 9.86 & 4.80 & 0.125 & 2.60 & 0 \\
\hline
\end{tabular}


between configurations II and III illustrate the transition between them. Finally, we remark that the shadows of KBHSH can have arbitrarily small sizes by considering solutions arbitrarily close to the BS curve in Fig. 1.

Remarks.-KBHSH can lead to qualitatively novel types of shadows in GR, as shown by shadows II and III. Even for KBHSH close to Kerr, their shadows are distinguishable from the latter, with the same asymptotic quantities, as illustrated by shadow I. Regardless of the astrophysical relevance of these solutions - which is unclear-they can yield new templates with small or large deviations from the Kerr shadows, hopefully of use for VLBI observations. An exhaustive analysis of KBHSH shadows spanning the space of solutions in Fig. 1, and at different observation angles, will be presented elsewhere, for producing such templates [32]. But the examples herein already raise a challenge to the parametrizations of deviations from Kerr suggested in the literature [7-11]: can they describe shadows with such large deviations?

Besides the peculiar shape of some of the shadows exhibited, this model has one general prediction: smaller observed shadows than those expected for Kerr BHs with the same asymptotic charges. Indeed, a "smaller" central $\mathrm{BH}$ seems a natural consequence of the existence of hair, carrying part of the total energy.

Finally, for the setup herein, the redshift, which depends only on the source's and $\mathcal{O}$ 's positions, is constant throughout the image and has been neglected.

We would like to thank T. Johannsen and V. Cardoso for discussions and correspondence and M. M. Soares for computational assistance. C. H. and E. R. acknowledge funding from the FCT-IF programme. H. R. is supported by Grant No. PD/BD/109532/2015 under the MAP-Fis Ph.D. program. This work was partially supported by the NRHEP295189 FP7-PEOPLE-2011-IRSES Grant, by FCT via Project No. PTDC/FIS/116625/2010, and by the CIDMA Strategic Project No. UID/MAT/04106/2013. Computations were performed at the Blafis cluster, in Aveiro University, and at the Laboratory for Advanced Computing, University of Coimbra.

[1] R. Narayan and J. E. McClintock, arXiv:1312.6698.

[2] E. Berti, E. Barausse, V. Cardoso, L. Gualtieri, P. Pani et al., arXiv:1501.07274.

[3] J. M. Bardeen, in Black Holes (Les Astres Occlus), edited by C. Dewitt and B.S. Dewitt (Gordon and Breach Science Publishers, New York, 1973), p. 215.

[4] H. Falcke, F. Melia, and E. Agol, Astrophys. J. 528, L13 (2000).

[5] A. E. Broderick, T. Johannsen, A. Loeb, and D. Psaltis, Astrophys. J. 784, 7 (2014).

[6] T. Johannsen and D. Psaltis, Astrophys. J. 718, 446 (2010).
[7] T. Johannsen and D. Psaltis, Phys. Rev. D 83, 124015 (2011).

[8] T. Johannsen, Phys. Rev. D 88, 044002 (2013).

[9] T. Johannsen, Phys. Rev. D 87, 124017 (2013).

[10] V. Cardoso, P. Pani, and J. Rico, Phys. Rev. D 89, 064007 (2014).

[11] L. Rezzolla and A. Zhidenko, Phys. Rev. D 90, 084009 (2014).

[12] L. Amarilla and E. F. Eiroa, Phys. Rev. D 85, 064019 (2012).

[13] A. Grenzebach, V. Perlick, and C. Lmmerzahl, Int. J. Mod. Phys. D 24, 1542024 (2015).

[14] J. W. Moffat, Eur. Phys. J. C 75, 130 (2015).

[15] C. A. R. Herdeiro and E. Radu, Phys. Rev. Lett. 112, 221101 (2014).

[16] F. Schunck and E. Mielke, Classical Quantum Gravity 20, R301 (2003).

[17] P. Amaro-Seoane, J. Barranco, A. Bernal, and L. Rezzolla, J. Cosmol. Astropart. Phys. 11 (2010) 002.

[18] B. Li, T. Rindler-Daller, and P. R. Shapiro, Phys. Rev. D 89, 083536 (2014).

[19] A. Suárez, V. H. Robles, and T. Matos, Astrophys. Space Sci. Proc. 38, 107 (2013).

[20] S. Doeleman, E. Agol, D. Backer, F. Baganoff, G. C. Bower, A. Broderick, A. Fabian, V. Fish, C. Gammie, P. Ho et al., in Astro2010: The Astronomy and Astrophysics Decadal Survey (National Academy, Washington, DC, 2009), Vol. 2010, p. 68.

[21] C. Herdeiro and E. Radu, Classical Quantum Gravity 32, 144001 (2015).

[22] A. Bohn, W. Throwe, F. Hbert, K. Henriksson, D. Bunandar, M. A. Scheel, and N. W. Taylor, Classical Quantum Gravity 32, 065002 (2015).

[23] The celestial sphere is centered around the BH or BS and is typically placed at twice the $R$ coordinate of $\mathcal{O}$. $\mathcal{O}$ is on the equatorial plane. See Sec. III in the Supplemental Material at http://link.aps.org/supplemental/10.1103/PhysRevLett.115 .211102 for other details.

[24] T. Johannsen, Astrophys. J. 777, 170 (2013).

[25] The circumferential radius is defined such that $2 \pi R=\oint d \varphi \sqrt{g_{\varphi \varphi}}$, where the metric component $g_{\varphi \varphi}$ is taken at a spacelike slice and on the equatorial plane, and $\partial_{\varphi}$ is the azimuthal Killing vector field.

[26] The subscript denotes the number of the solution in Fig. 1 or other relevant information: ER1/ER2 = first/second Einstein ring, $\mathrm{LR}=$ light ring, and $\mathrm{BB} 1=$ first back bending. The superscript denotes the branch. The branch changes after each backbending in the $M-w$ plot.

[27] In both the spherical and rotating BS cases, it is actually a pair of light rings that appears.

[28] V. Cardoso, L. C. B. Crispino, C. F. B. Macedo, H. Okawa, and P. Pani, Phys. Rev. D 90, 044069 (2014).

[29] C. A. R. Herdeiro and E. Radu, arXiv:1505.04189.

[30] A. Yumoto, D. Nitta, T. Chiba, and N. Sugiyama, Phys. Rev. D 86, 103001 (2012).

[31] S. Abdolrahimi, R. B. Mann, and C. Tzounis, Phys. Rev. D 91, 084052 (2015).

[32] P. Cunha, C. Herdeiro, E. Radu, and H. Rúnarsson (to be published). 